\title{
NUMERICAL SIMULATIONS OF MULTIPLE SHOCK WAVE BOUNDARY LAYER INTERACTIONS
}

\author{
K. BOYCHEV ${ }^{1}$, G.N. BARAKOS ${ }^{2}$ AND R. STEIJL ${ }^{3}$ \\ ${ }^{1} \mathrm{PhD}$ Student \\ CFD Lab, University of Glasgow, G128QQ \\ k.boychev.1@research.gla.ac.uk \\ ${ }^{2}$ Professor \\ CFD Lab, University of Glasgow, G128QQ \\ g.barakos@glasgow.ac.uk \\ ${ }^{3}$ Senior Lecturer \\ CFD Lab, University of Glasgow, G128QQ \\ r.steij1@glasgow.ac.uk
}

Key words: multiple shock wave boundary layer interactions, MSWBLI, shock train, pseudo shock, non-linear EVMs, high-speed intakes

\begin{abstract}
A parametric study of Multiple Shock Wave Boundary Layer Interactions is presented in this paper. All results were obtained using the Computational Fluid Dynamics Solver of Glasgow University. Such interactions often occur in high-speed intakes, depending on the state of the upstream boundary layer, and can adversely affect the performance of the intake. First simulations of multiple shock wave boundary layer interaction in a rectangular duct were performed and compared to the experiments followed by simulations at different Mach and Reynolds numbers and flow confinement levels. The results showed that Reynolds-stress based turbulence models are better suited than linear turbulence models in predicting the interaction. The employed Explicit Algebraic Reynolds Stress Model showed good agreement for the corner and centreline separations and resulted only in a small underprediction of the wall pressure. Flow distortion and total pressure recovery efficiency metrics were defined and evaluated for each interaction. Lower upstream Mach number and/or lower levels of flow confinement were required to achieve higher total pressure recoveries and lower flow distortion levels.
\end{abstract}

\section{INTRODUCTION}

High-speed intakes use shock waves to decelerate (compress) the oncoming flow. Within the intake, shock waves interact with the boundary layer. This phenomenon is referred to as a shock wave boundary layer interaction (SWBLI). SWBLIs impact the performance of the high-speed intake, in particular, the flow distortion (FD) and total pressure (TPR) recovery. For maximum efficiency, the former parameter must be low and the latter high. Since SWLBIs are affected by the upstream conditions, sometimes the state of the boundary layer can promote the formation of multiple SWBLIs or MSWBLIs. As the name suggests a multiple SWBLI is a series of consecutive shocks interacting with the boundary layer, 
observed in internal flows. The upstream Mach number $M_{r}$ and level of flow confinement $\delta_{r} / h$ are the parameters promoting the formation of MSWBLIs where $\delta_{r}$ is the thickness of the boundary layer and $h$ is the duct half-height. Higher values for both often result in MSWBLIs. The effect of increased flow confinement is however less dominant at higher Mach numbers. MSWBLIs are also referred to as shock trains or pseudo-shocks. Although the terms are used interchangeably at times, the term shock train refers to the region where shocks are present and the pseudo- shock refers to the entire region of pressure rise. The region immediately downstream of the shock train region is termed the mixing region. In this region supersonic/subsonic flow mixing is present. Figure 1 shows a schematic of an MSWBLI inside a rectangular duct.

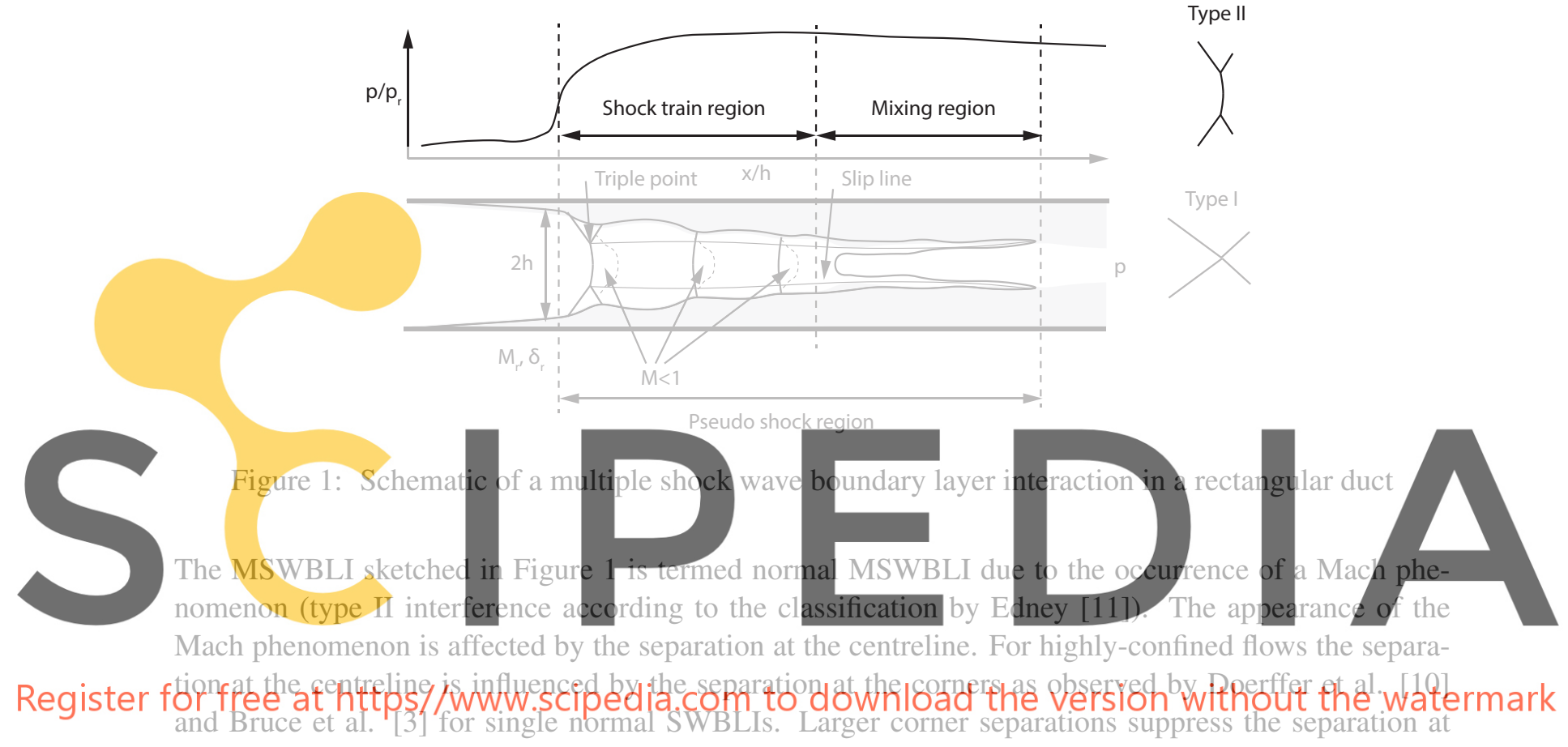

the centreline and result in the appearance of a Mach phenomenon. Larger separation at the centreline reduces the Mach phenomenon and eventually leads to a type I interference. Such interactions are exclusively observed at higher Mach numbers. For the type II interference (Mach phenomenon is present) a secondary shear layer emanates from the triple (or bifurcation) point. Flow near the secondary shear layer remains supersonic for longer distances downstream. These regions of supersonic flow are termed "supersonic tongues". The transition from a single to multiple type II interference and to multiple type I interference is accompanied by a significant increase in the interaction length. Being able to predict such interactions with a reasonable degree of confidence is important in the context of high-speed intake design, therefore a robust numerical method capable of predicting these interactions is required. The method and the employed models must be able to accurately account for secondary flows and resolve the corner and centreline separations. Current efforts in LES and hybrid RANS/LES, such as SAS and DES in its different formulations, still require reduction of the Reynolds number by an order of magnitude or rely on linear eddy-viscosity based models that suffer from the inability to account for the secondary flows arising near corners. The objective of this paper is to present validation and verification of an MSWBLI interaction and investigate the effect of Mach number $M_{r}$, Reynolds number $R e_{h}$, and level of 
flow confinement $\delta_{r} / h$ using the k- $\omega$ EARSM turbulence model. Section 2 and 3 discuss the numerical method and setup. The results are presented in section 4 as follows: the validation cases are discussed first followed by the parametric cases.

\section{NUMERICAL METHOD}

Numerical simulations of the steady flowfields have been performed using the Helicopter Multi-Block (HMB3) flow solver $[17,16]$. The flow solver is a three-dimensional, fully implicit, structured, multiblock Unsteady Reynolds Averaged Navier-Stokes (URANS) code solving the URANS equations in integral form using the Arbitrary Lagrangian-Eulerian (ALE) formulation for time-dependent domains, which may include moving boundaries. The equations are discretised using a cell-centred finite volume approach. The spatial discretisation of these equations leads to a set of ordinary differential equations in time

$$
\frac{d}{d t}\left(\mathbf{W}_{i, j, k} V_{i, j, k}\right)=-\mathbf{R}_{i, j, k}\left(\mathbf{W}_{i, j, k}\right),
$$

where $i, j, k$ represent the cell index, $\mathbf{W}$ and $\mathbf{R}$ are the vectors of conservative flow variables and flux residual respectively, and $V_{i, j, k}$ is the volume of the cell $i, j, k$. The Osher [15] approximate Riemann solver is used to evaluate the convective fluxes while the viscous terms are discretised using a second-order central difference scheme. The Monotonic Upwind Scheme for Conservation Laws (MUSCL) approach

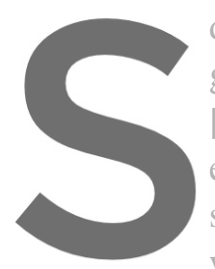
developed by van Leer gradients are encountered [19] is activated to avoic employed to perform the state simulations where th
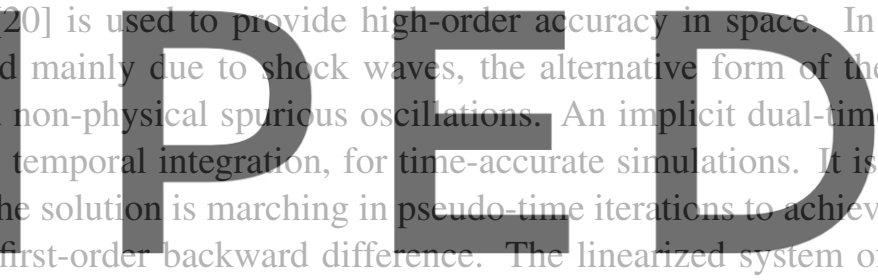
which is solved using : using the Generalised Conjugate Gradient method with a Block Incomplete Lower-Upper (BILU) fac-

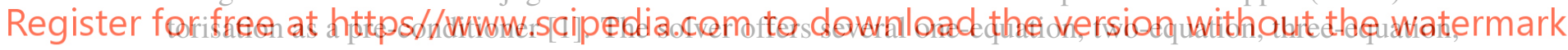
and four-equation turbulence models. In addition Large-Eddy Simulation (LES), Detached-Eddy Simulation (DES), Delayed-Detached-Eddy Simulation (DDES), Improved-Delayed-Detached-Eddy Simulation (IDDES), and Scale-Adaptive-Simulation (SAS) are also available. The fully-turbulent $k-\omega$ EARSM [21, 13] turbulence model is used due to its good agreement with experimental data for this case.

\section{NUMERICAL SETUP}

The MSWBLI experiment by Carroll and Dutton $[4,5,6,7,8,9]$ is targeted for comparison. In the experiment, a $753.8 \mathrm{~mm}$ long rectangular test section with constant width of $2 w=76.2 \mathrm{~mm}$ and a divergence angle of $0.13 \mathrm{deg}$ was used. LDV measurements were performed between $x=x_{r}=264.8 \mathrm{~mm}$ and $x=664.8 \mathrm{~mm}$ over variable intervals where $x_{r}$ designates the onset of the interaction. From the boundary layer measurements the height of the rectangular test section at $x_{r}=264.8 \mathrm{~mm}$ was $2 h_{r}=33.75 \mathrm{~mm}$ $\left(\delta_{r} / h=0.32, \delta_{r}=5.4 \mathrm{~mm}\right)$. The upstream Mach number was $M_{r}=1.61$ and the unit Reynolds number was $R e=3.0 \times 10^{7} \mathrm{~m}^{-1}$. The Reynolds number based on the half-height of the duct at $x_{r}=264.8$ is, 
therefore, $R e_{h} \approx 5.06 \times 10^{5}$. A schematic of the experimental setup is shown in Figure 2 below.

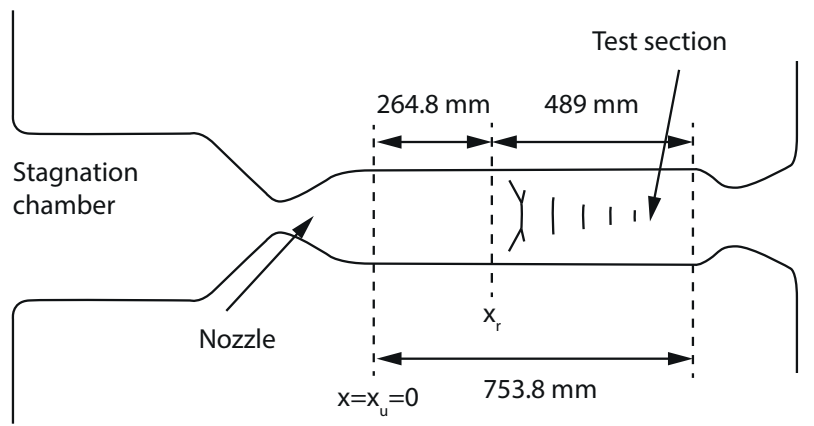

Figure 2: Schematic of the experimental setup of the MSWBLI experiment by Carroll \& Dutton [4, 9].

\subsection{NUMERICAL DOMAIN, GRIDS AND BOUNDARY CONDITIONS}

\section{A numerical domain of length $L_{x} / h=46.32$ was used for the shock train simulations of the experiment}

by Carroll et al. [4]. The domain was made dimensionless by the duct half-height at $x / h=0(h=16.275$ $\mathrm{mm})$. Figure 3 shows a 2D schematic of the employed numerical domain.
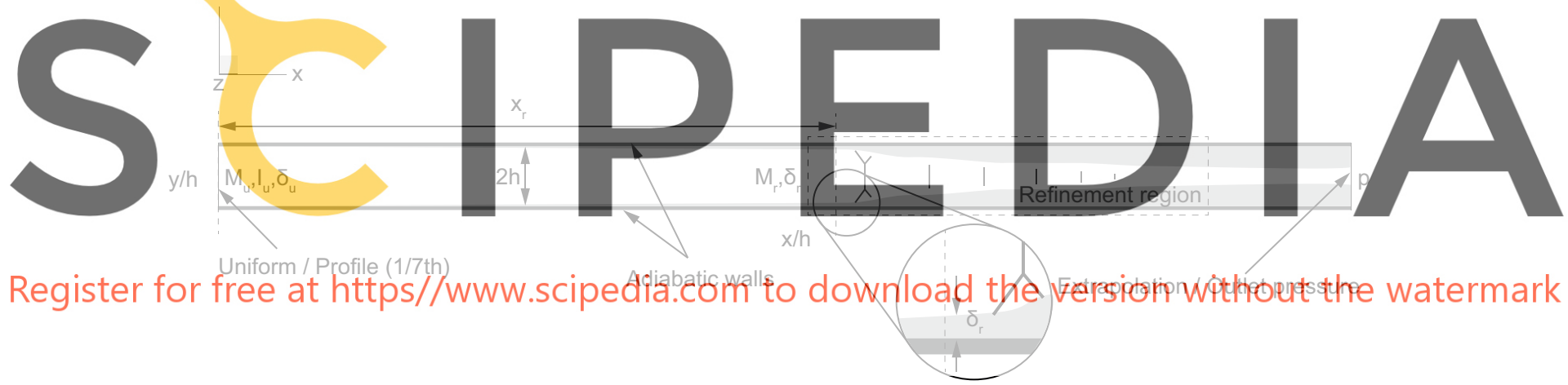

Figure 3: 2D schematic of the numerical domain and boundary conditions for the shock train simulations.

At the inlet, a uniform profile for the flow variables was specified. The turbulence intensity and eddy viscosity ratio were set to $I_{u}=0.01(1 \%)$ and $\frac{\mu_{t}}{\mu}=10$. The Mach number at the inlet was higher than the Mach number before the start of the interaction $M_{u}>M_{r}$ to take into account the area reduction due to the boundary layer growth. The adjustments of $M_{u}$ and the outlet pressure $p$ allowed to match the upstream and downstream conditions as closely as possible. At the outlet, a first-order extrapolation was performed except where the flow is subsonic. There, the outlet pressure $p$ was specified. Adiabatic wall boundary conditions were used for all walls. Symmetry boundary condition was applied at the $x-z$ and $x-y$ planes since previous investigations showed that the flow exhibits symmetry. This resulted in simulating only a quarter of the numerical domain. Figure 4 (a) shows grid B (mirrored cross the $z-x$ and $y-x$ planes). The numerical domain is outlined with a solid red line. Also shown in Figure 4 (b) is 
the cross-section of grid B at $x / h=0$. As observed from Figure 3 all grids feature a refinement region in the streamwise $(x / h)$ direction to resolve the pseudo-shock. For grid B the streamwise refinement region extends farther upstream to allow movement of the pseudo-shock due to changes in the inlet and outlet conditions. For all grids the first grid point was located at $y^{+}<<1$. The average $y^{+}$at the wall for grid A3 was $\approx 0.1$. Table 1 lists the grid parameters.
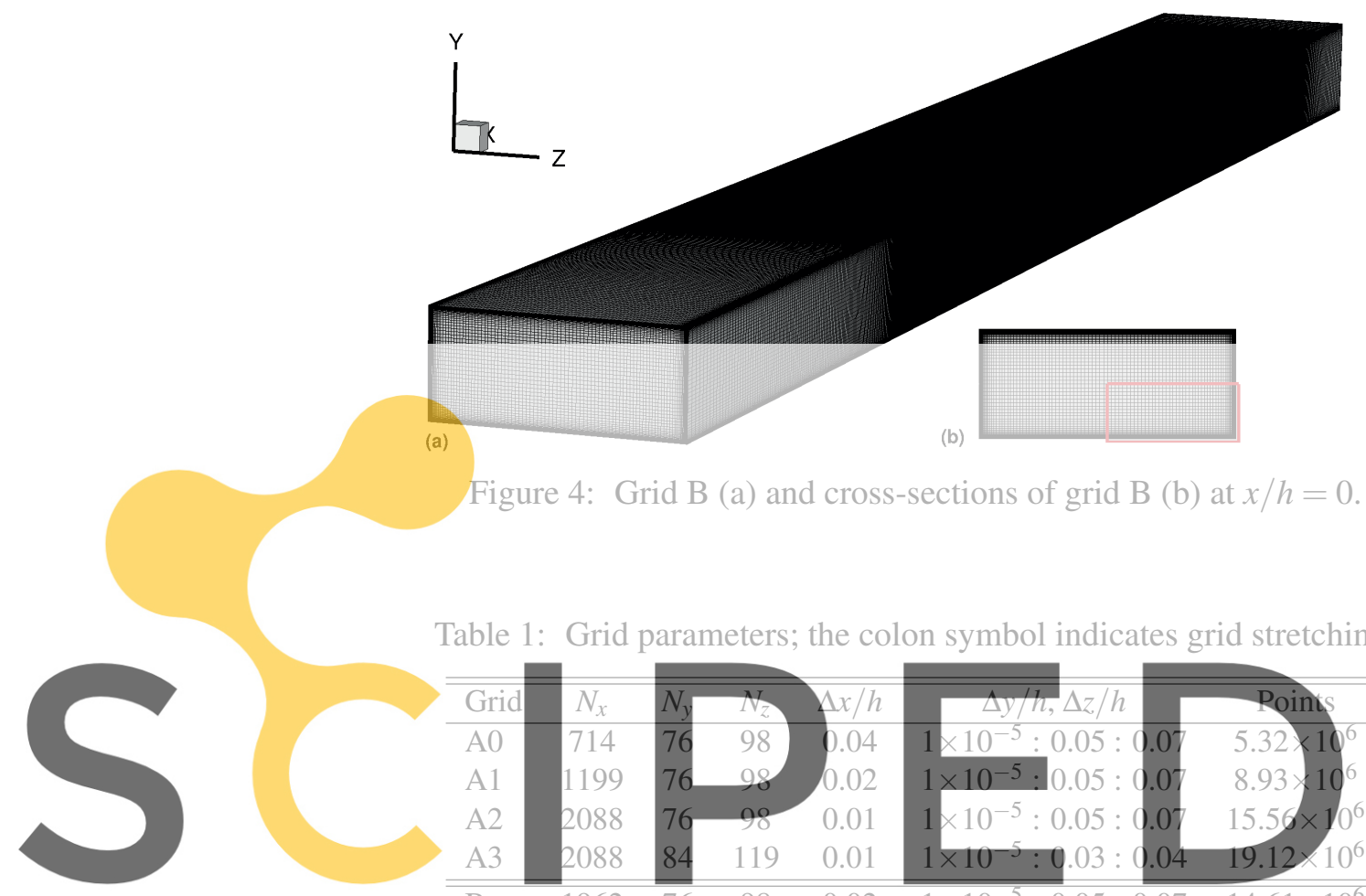

Table 1: Grid parameters; the colon symbol indicates grid stretching.
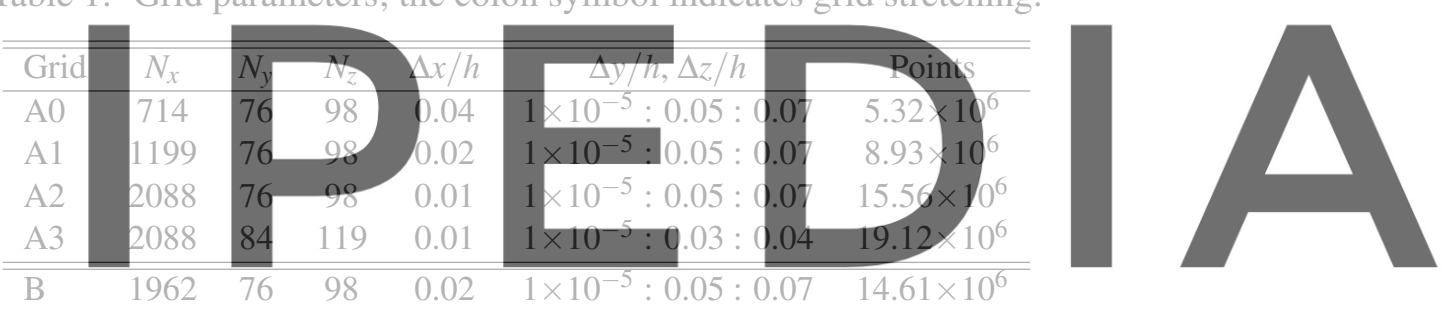

Register for free at https//www.scipedia.com to download the version without the watermark

\subsection{NUMERICAL CASES}

A total of 9 MSWBLI simulations were performed. Table 2 summarises the simulation parameters and results. First three simulations investigate the effect of grid refinement and the remaining - the effect of varying Mach number, $M_{r}$, Reynolds number, $R e_{h}$, and flow confinement, $\delta / h$. All simulations were initialized with an inviscid shock with a pre-shock Mach number of $M_{u}$ at the end of the domain. Approximately $10^{5}$ implicit steps at CFL of 4 were required for the solution to converge to at least 5 orders of magnitude in the flux residuals. 
Table 2: Simulation parameters.

\begin{tabular}{lccccccccc}
\hline \hline Case & $\mathrm{Grid}$ & $R_{h}$ & $M_{u}$ & $M_{r}$ & $\delta_{r} \mathrm{~mm}$ & $\delta_{r} / h$ & $x_{r} / h$ & $p / p_{u}$ & $p / p_{r}$ \\
\hline Reference & $\mathrm{A} 1$ & $4.9 \times 10^{5}$ & 1.690 & 1.627 & 5.14 & 0.32 & 26.4 & 2.4776 & 2.3337 \\
Reference & $\mathrm{A} 2$ & $4.9 \times 10^{5}$ & 1.690 & 1.616 & 5.00 & 0.31 & 26.0 & 2.4776 & 2.2320 \\
Reference & $\mathrm{A} 3$ & $4.9 \times 10^{5}$ & 1.690 & 1.616 & 4.63 & 0.28 & 25.7 & 2.4776 & 2.2309 \\
\hline \hline Reference & $\mathrm{B}$ & $4.9 \times 10^{5}$ & 1.690 & 1.619 & 5.02 & 0.31 & 26.1 & 2.4775 & 2.2299 \\
Lowest $M_{u}$ & $\mathrm{~B}$ & $4.9 \times 10^{5}$ & 1.490 & 1.455 & 2.32 & 0.14 & 10.8 & 2.2379 & 2.1082 \\
Lower $M_{u}$ & $\mathrm{~B}$ & $4.9 \times 10^{5}$ & 1.590 & 1.536 & 3.12 & 0.19 & 15.0 & 2.4775 & 2.2883 \\
Lower $R e_{h}$ & $\mathrm{~B}$ & $4.9 \times 10^{4}$ & 1.690 & 1.572 & 4.99 & 0.31 & 15.3 & 2.4775 & 2.0781 \\
Lowest $\delta_{r} / h$ & $\mathrm{~B}$ & $4.9 \times 10^{5}$ & 1.690 & 1.643 & 2.46 & 0.15 & 11.6 & 2.8609 & 2.6936 \\
Lower $\delta_{r} / h$ & $\mathrm{~B}$ & $4.9 \times 10^{5}$ & 1.690 & 1.644 & 3.22 & 0.20 & 15.6 & 2.7818 & 2.5543 \\
\hline Experiment $[4]$ & & & $\mathbf{1 . 6 1 0}$ & 5.40 & 0.32 & 0 & & 2.2309 & \\
\hline \hline
\end{tabular}

\section{RESULTS AND DISCUSSION}

\subsection{VALIDATION CASES}

The effect of grid refinement, the inclusion/exclusion of spanwise effects and the effect of turbulence modelling on the MSWBLI interaction by Carroll et al. [4] were investigated previously by Boychev et al. [2]. In the aforementioned work two additional MSWBLI cases, often used in the literature, were simulated - the Mach 2 MSWBLI case by Sun et al. [18] and the scale resolving simulation of Fiévet et

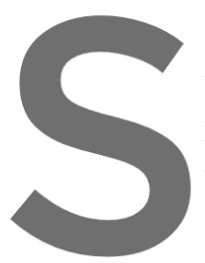
al. [12] representing the three cases considered the moderate sizes $\left(8 \times 10^{6}\right.$ the wall pressure for the
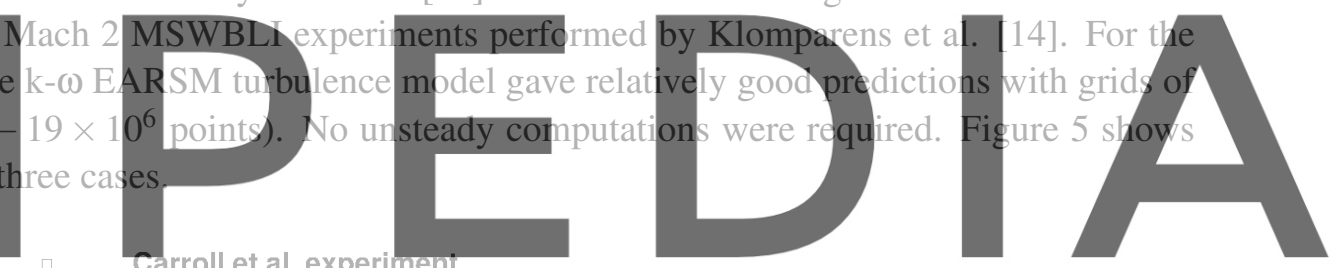

Register for free at https//www. scipedial.cơneripendownload the version without the watermark

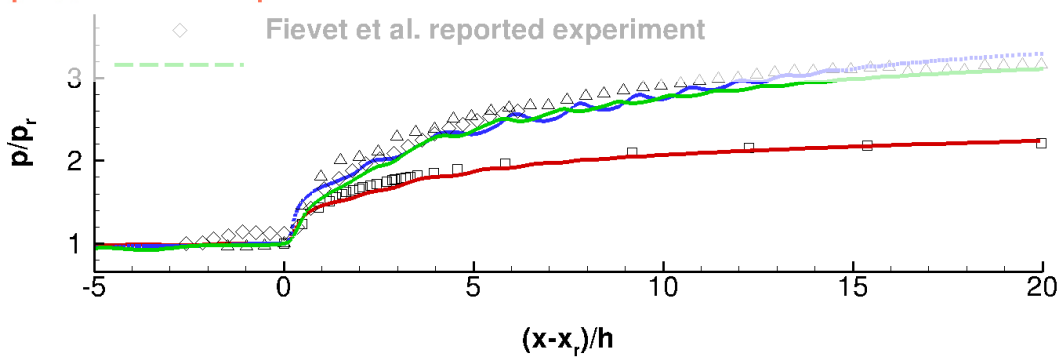

Figure 5: Wall pressure for the cases of Carroll et al. [4], Sun et al. [18], and Fiévet et al. [12]; numerical simulations are represented by lines

The Scale Adaptive Simulation (SAS) approach was tested for the same MSWBLI case of Carroll et al. [4] and results are shown in figure 6 where the shock train is visible. For the parametric study, the work employed the same k- $\omega$ EARSM turbulence model. 


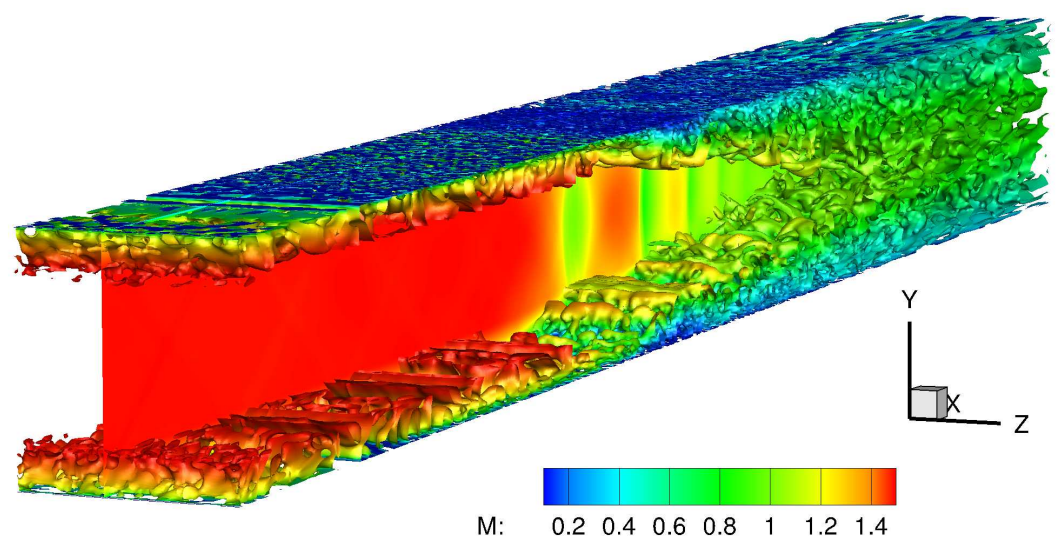

Figure 6: Wall pressure for the cases of Carroll et al. [4], Sun et al. [18], and Fiévet et al. [12]; numerical simulations are represented by lines

\subsection{PARAMETRIC CASES}

The parametric cases featuring a smaller pre-shock Mach number $M_{r}$ or smaller level of confinement $\delta_{r} / h$ shared similar separation patterns. For he lowest $M_{\mu}$, lower $M_{\psi}$, lowest $\delta_{r} / h$, and lower $\delta_{r} / h$ cases large corner separations and centreline separations were observed. The shock trains were considerably shorter, and the interactions featured a type II interference. The pre-shock Mach number was observed For maximum total pressure recovery and minimum flow distortion, a shock train featuring a type II interference is preferred. Table 3 lists the total pressure recovery and flow distortion for all cases and

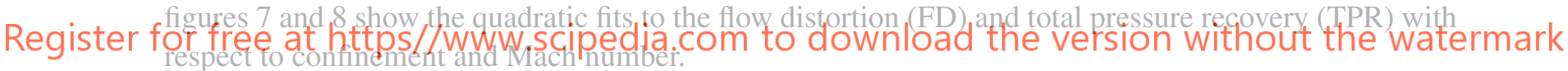

Table 3: Total pressure recovery and flow distortion.

\begin{tabular}{lccc}
\hline \hline Case & $T P R_{\text {ref }}$ & $T P R$ & $F D$ \\
\hline Reference & 0.860 & 0.758 & 0.644 \\
Lowest $M_{u}$ & 0.933 & 0.869 & 0.501 \\
Lower $M_{u}$ & 0.899 & 0.825 & 0.516 \\
Lower $R e_{h}$ & 0.860 & 0.763 & 0.655 \\
Lowest $\delta_{r} / h$ & 0.860 & 0.798 & 0.524 \\
Lower $\delta_{r} / h$ & 0.860 & 0.783 & 0.573 \\
Reduced $w / h$ & 0.860 & 0.756 & 0.649 \\
\hline \hline
\end{tabular}

From the table and figures, it is observed that a low pre-shock Mach number or small levels of flow confinement is required to maximize the TPR and minimize the FD. 


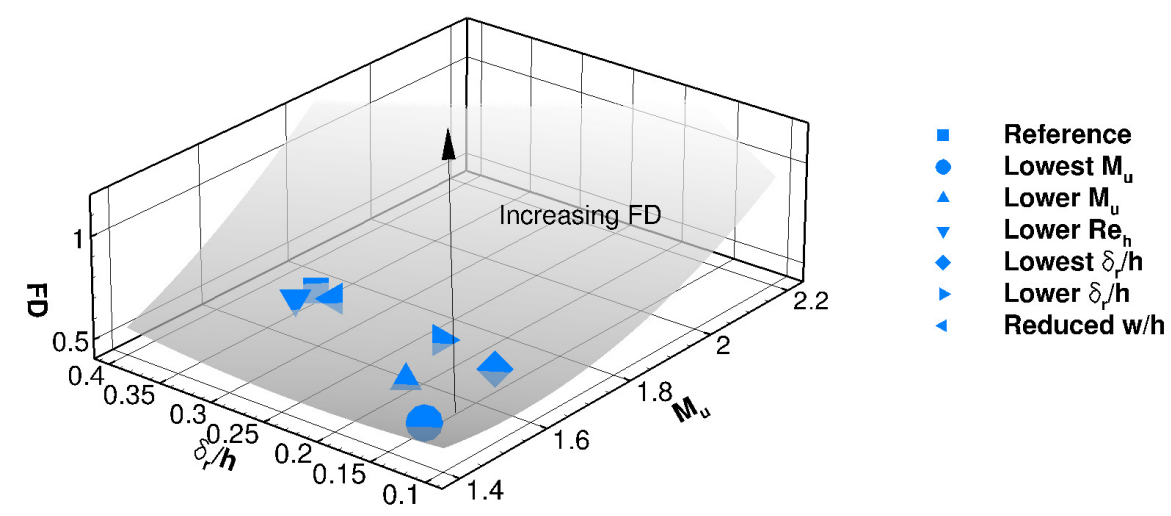

Figure 7: Flow distortion versus Mach number and flow confinement; a quadratic surface fit is shown.

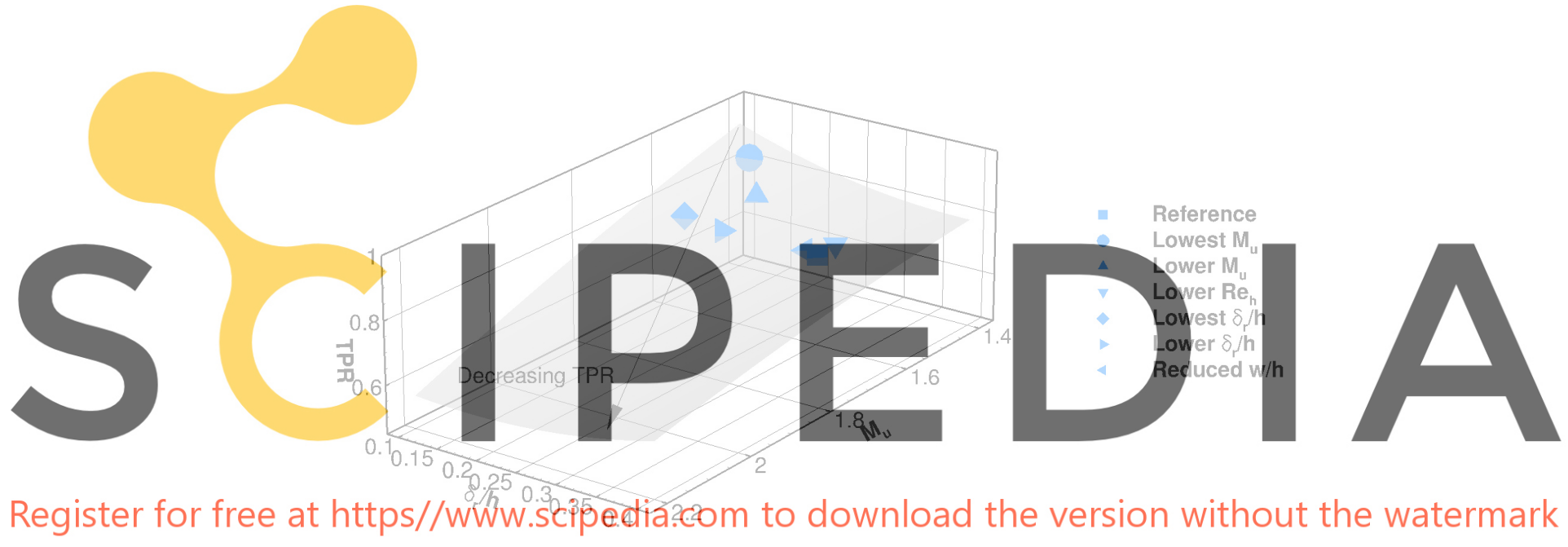

Figure 8: Total pressure recovery versus Mach number and flow confinement; a quadratic surface fit is shown.

\section{CONCLUSIONS}

This work shows that a non-linear eddy viscosity based model can offer improved predictions for flows featuring shock trains. Three different cases having different Mach and Reynolds numbers were considered. From the analysis of the separation patterns and the wall shear stress visualised with friction lines just above the surface, it was observed that the non-linear model results in smaller corner separations and large separation at the centreline. The parametric studies investigating the effect of Mach number, Reynolds number, and level of flow confinement showed that for increased total pressure recovery and decreased flow distortion lower pre-shock Mach numbers and confinement levels are required. Maximizing the total pressure recovery and minimizing the flow distortion is the primary objective in the context of high-speed intake design. 


\section{FUTURE WORK}

Future work includes scale resolving simulations employing hybrid RANS/LES methodology and simulations of more realistic geometries (high-speed intakes).

\section{REFERENCES}

[1] O. Axelsson. Iterative Solution Methods. Cambridge University Press, 1994.

[2] K. Boychev, G.N. Barakos, and R. Steijl. Flow physics and sensitivity to RANS modelling assumptions of a multiple shock wave/turbulent boundary layer interaction. Aerospace Science and Technology, 97(1), 2020.

[3] P.J.K Bruce, Babinsky H., B. Tartinville, and C. Hirsch. Corner effect and asymmetry in transonic channel flows. AIAA Journal, 49(11):2382-2392, 2011.

[4] B.F. Carroll. Numerical and Experimental Investigation of Multiple Shock Wave/Turbulent Boundary Layer Interactions in a Rectangular Duct. PhD thesis, University of Illinois at UrbanaChampaign, Urbana, IL, 1988.

[5] B.F. Carroll and J.C. Dutton. An LDV investigation of a multiple normal shock wave/turbulent boundary layer interaction. In 27th Aerospace Sciences Meeting, 1989.

[6] B.F. Carroll and J.C. Dutton. Characteristics of multiple shock wave/turbulent boundary-layer

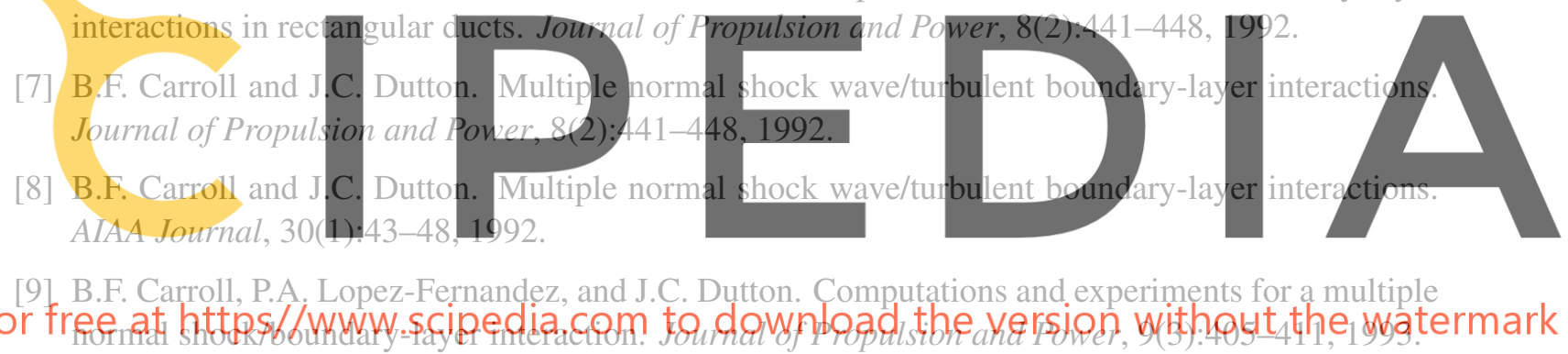

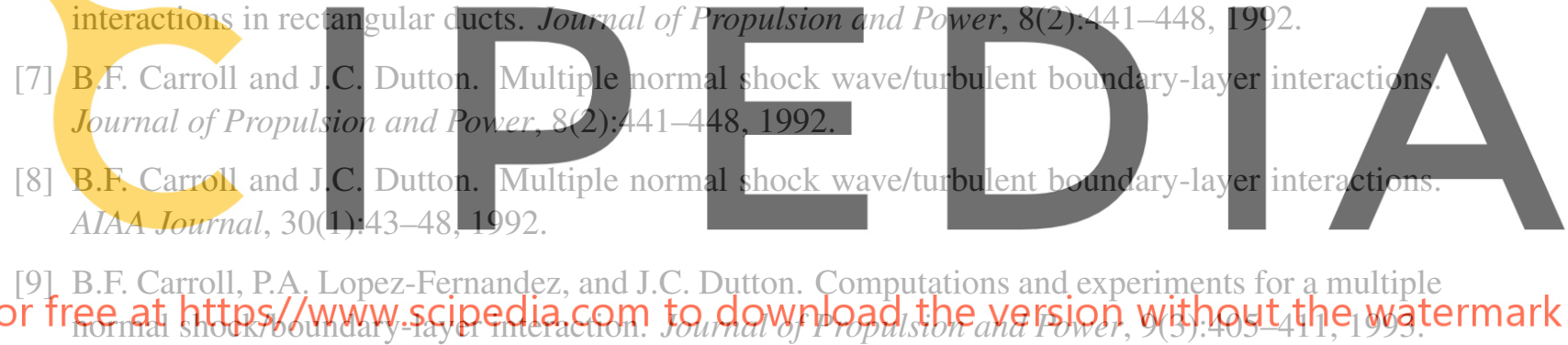

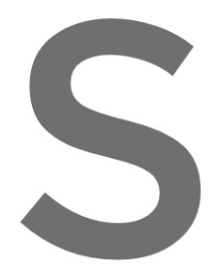

[10] P. Doerffer, C. Hirsch, J.P. Dussauge, H. Babinsky, and G.N. Barakos. Unsteady Effects of Shock Wave Induced Separation: 220 (Notes on Numerical Fluid Mechanics and Multidisciplinary Design). Springer, 1 edition, 2011.

[11] B. Edney. Anomalous heat transfer and pressure distributions on blunt bodies at hypersonic speeds in the presence of an impinging shock. Technical report, Aeronautical Research Institute Sweden, 1968.

[12] R. Fiévet, H. Koo, V. Raman, and A.H. Auslender. Numerical investigation of shock-train response to inflow boundary-layer variations. AIAA Journal, 55(9):2888-2900, 2017.

[13] A. Hellsten. New advanced k-omega turbulence model for high-lift aerodynamics. AIAA Journal, 43(9):1857-1869, 2005.

[14] R. Klomparens, J. Driscoll, and M. Gamba. Unsteadiness characteristics and pressure distribution of an oblique shock train. In 53rd AIAA Aerospace Sciences Meeting, 2015.

[15] S. Osher and S. Chakravarthy. Upwind schemes and boundary conditions with applications to euler 
equations in general geometries. Journal of Computational Physics, 50(3):447-481, 1983.

[16] R. Steijl and G. N. Barakos. Sliding mesh algorithm for CFD analysis of helicopter rotor-fuselage aerodynamics. International Journal for Numerical Methods in Fluids, 58(5):527-549, 2008.

[17] R. Steijl, G. N. Barakos, and K. Badcock. A framework for CFD analysis of helicopter rotors in hover and forward flight. International Journal for Numerical Methods in Fluids, 51(8):819-847, 2006.

[18] L.Q. Sun, H. Sugiyama, Mizobata K., T. Hiroshima, and A. Tojo. Numerical and experimental study of the mach 2 pseudo-shock wave in a supersonic duct. 2005.

[19] G. D. van Albada, B. van Leer, and W. W. Roberts. A comparative study of computational methods in cosmic gas dynamics. Astronomy and Astrophysics, 108(1):76-84, 1982.

[20] B. van Leer. Towards the ultimate conservative difference scheme. V. A second-order sequel to godunov's method. Journal of Computational Physics, 32(1):101-136, 1979.

[21] S. Wallin and A.V. Johansson. An explicit algebraic reynolds stress model for incompressible and compressible turbulent flows. Journal of Fluid Mechanics, 403:89-132, 2000.
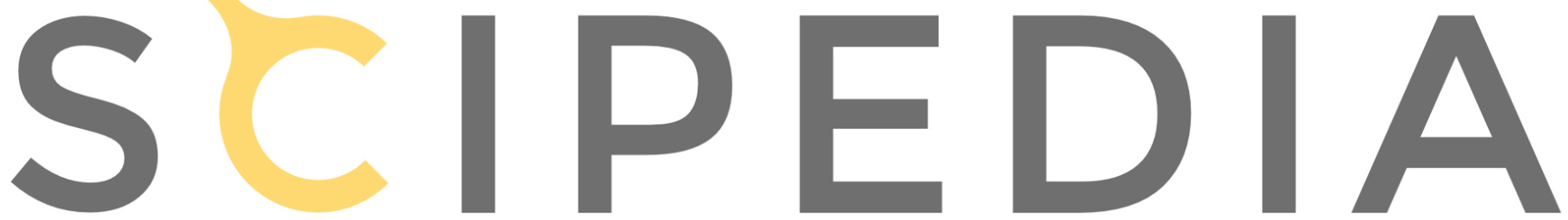

Register for free at https//www.scipedia.com to download the version without the watermark 